\title{
Clinical application of 3D imaging for assessment of treatment
}

\section{outcomes}

\author{
Lucia H.C. Cevidanes, DDS, MS, PhDa, Ana Emilia Figueiredo Oliveira, DDS, MS, $\mathbf{P h D}^{\mathrm{b}}$, \\ Dan Grauer, DDS, MS ${ }^{\mathrm{C}}$, Martin Styner, $\mathbf{P h D}^{\mathrm{d}}$, and William R. Proffit, DDS, $\mathbf{P h D}^{\mathrm{e}}$ \\ ${ }^{a}$ Assistant Professor, Department of Orthodontics, School of Dentistry, University of North \\ Carolina \\ bProfessor, Department of Dentisty-1, Federal University of Maranhão, Brazil \\ cPhD Student, Department of Orthodontics, School of Dentistry, University of North Carolina \\ ${ }^{\mathrm{d}}$ Assistant Professor, Departments of Psychiatry and Computer Science, University of North \\ Carolina
}

eKenan Professor, Department of Orthodontics, School of Dentistry, University of North Carolina

\begin{abstract}
This paper outlines the clinical application of CBCT for assessment of treatment outcomes, and discusses current work to superimpose digital dental models and 3D photographs. Superimposition of CBCTs on stable structures of reference now allow assessment of 3D dental, skeletal and soft tissue changes for both growing and non-growing patients. Additionally, we describe clinical findings from $\mathrm{CBCT}$ superimpositions in assessment of surgery and skeletal anchorage treatment.
\end{abstract}

\section{Introduction}

Assessment of treatment outcomes using $\mathrm{CBCT}$ has the potential to unravel the interactions between the dental, skeletal and soft tissue components that underpin the response to treatment. ${ }^{1}$ Although differentiating dentofacial changes caused by treatment from those induced by growth is still not possible with either $2 \mathrm{D}$ or $3 \mathrm{D}$ superimposition methods, ${ }^{2-4} 3 \mathrm{D}$ superimpositions reveal areas of bone displacement and remodeling. $5-6$

The goal of 3D superimposition of serial images is to understand how changes in size and shape and shifts in relative positions of skeletal and soft tissue facial components contribute to orthodontic/orthopedic or surgical treatment changes. Such understanding has the potential to improve our interpretations of variations in patient response to treatment. Before the application of CBCT technology can be translated into improved treatment outcomes assessment, it is necessary to understand the $3 \mathrm{D}$ registration and superimposition processes.

The first step in the registration process is to determine which structures will be used as a stable reference. The displacement, change in shape or in size will be later described relative

(C) 2010 Elsevier Inc. All rights reserved.

Corresponding Author: Dr. Lucia H.C. Cevidanes, Department of Orthodontics, UNC School of Dentistry, 201 Brauer Hall, CB7450, Chapel Hill, NC 27599, Cell: (919) 357-8603, Fax: (919) 843-8864, cevidanl@ dentistry.unc.edu.

Publisher's Disclaimer: This is a PDF file of an unedited manuscript that has been accepted for publication. As a service to our customers we are providing this early version of the manuscript. The manuscript will undergo copyediting, typesetting, and review of the resulting proof before it is published in its final citable form. Please note that during the production process errors may be discovered which could affect the content, and all legal disclaimers that apply to the journal pertain. 
to these structures. In 2D cephalometrics, the cranial base often is used for superimpositions because it shows minimal changes after neural growth is completed. In 3D image analysis, registration can be based on choice of stable surfaces or landmarks. While landmark location in 2D is hampered by identification of hard and soft tissues on $\mathrm{x}$-rays due to the superimposition of multiple structures, locating 3D landmarks on complex curving structures is significantly more difficult. As Bookstein ${ }^{7-8}$ noted, there are no suitable operational definitions for craniofacial landmarks in the 3 planes of space (coronal, sagittal, and axial). In the context of facial changes, superimposition should not rely on landmark identification nor on best-fit techniques on structures that may have changed between acquisitions. This paper describes the use of a fully automated voxel-wise rigid registration at the cranial base and application of 3D superimposition methods to evaluate dental, skeletal and soft tissue changes. These assessments will improve our understanding of softand hard-tissue facial form and changes with growth and treatment; they will also facilitate the creation of normative databases and predictive algorithms.

The major strength of this superimposition method is that registration does not depend on the precision of the 3D surface models. The cranial base models are only used to mask anatomic structures that change with growth and treatment. The registration procedure actually compares voxel by voxel of gray level CBCTs images, containing only the cranial base, and calculates the rotation and translation parameters between the 2 time point images.

\section{CBCT SUPERIMPOSITIONS: ASSESSMENT OF TREATMENT/GROWTH CHANGES USING SURFACE REGISTRATION ON THE CRANIAL BASE}

\section{Image Acquisition}

Cone-beam CT (CBCT) equipment specialized for maxillofacial imaging offers a relatively low-dose and convenient way to assess three-dimensional changes in facial morphology for both growing and non-growing subjects. CBCT scans presented in this paper were acquired with either the iCat (Imaging Sciences International,Hatfield, PA) $16 \times 22 \mathrm{~cm}$ field of view, or NewTom 3G (Aperio Services LLC, Sarasota, FL) 12-inch field of view CBCT scanners including the entire facial anatomy. The images were reformatted ${ }^{9}$ to yield a voxel size of $0.5 \mathrm{~mm}$, and then cropped to facilitate image analysis. Experimental protocols were approved by the University of North Carolina (UNC) Institutional Review Board.

\section{Image Analysis}

Analysis of serial CBCT images to evaluate changes over time includes four steps: (1) model construction, (2) image registration, (3) transparency overlay, and (4) quantitative measurement.

(1) Construction of virtual 3D surface models (Figure 1)-Surface models are created using ITK-SNAP open-source software. ${ }^{10}$ This construction of surface models differs from currently available commercial software that display 3D projections of the face (3D rendering).

(2) Image registration (Figure 2)-Image registration is a core technology for many imaging tasks. According to the transformation applied to the images, registration procedures can be classified into two main groups: rigid and nonrigid. The transformation involved in a rigid registration procedure includes translation and rotation, while that of a nonrigid registration includes translation, rotation, scale and affine properties. The two obstacles to widespread clinical use of nonrigid (elastic and deformable) registration are computational cost and quantification difficulties as the 3D models are deformed. Nonrigid registration would be required to create a composite of several different jaw shapes to guide 
the construction of template or standard, normal 3D surface models. To evaluate longitudinal changes, rigid registration is acceptable.

Using rigid registration and Imagine Software ${ }^{11}$ the authors mask anatomical structures that have changed with growth or treatment and then perform a fully automated, voxel-wise, rigid registration at the cranial base.

For superimposition of CBCT scans of subjects whose growth is complete, registration of virtual 3D surface models is done using the whole surface of the cranial base (Figure 2A). For superimposition of CBCT scans of growing individuals, the anterior cranial fossae and the ethmoid bone surfaces can be used in the registration procedure given that the growth of these structures is completed in early childhood. ${ }^{12}$ The anterior cranial base of the CBCT images is used as the reference for superimposing different time points (Figure 2B). A fully automated registration of 3D surface models that uses the Imagine Software to mask anatomical structures changed with growth and treatment was used. The Imagine Software ${ }^{11}$ computes the rigid registration (translation and rotation, Figure 2C) that optimally aligns the before- and after-treatment gray level CBCT datasets with subvoxel accuracy at the cranial base.

(3) Transparency overlay (Figure 3)-Once both images from different time points are registered, they share the same coordinate system. The next step in the analysis involves overlaying the registered 3D model surfaces with another tool, CMF software (Maurice Müller Institute, Bern, Switzerland). ${ }^{13}$ This tool allows different degrees of transparencies to visually assess the boundaries of the maxillo-mandibular structures between superimposed models at two different time points. The location, magnitude, and direction of dental, bone and soft tissue displacements can be clearly identified.

(4) Quantitative measurements-The CMF application software is then used to measure overall facial changes. ${ }^{13}$ While evaluating sequential 3D models, precise quantitative measurement is required to assess vertical, transverse, and anteroposterior soft tissue changes that accompany growth and response to treatment. Landmark-based measurements present errors related to landmark identification. Andresen et al. ${ }^{14}$ and Mitteroecker et al. ${ }^{15}$ proposed the use of "semilandmarks," or landmarks plus vectors and tangent planes that define their location, but information from the whole curves and surfaces must also be included. Gerig et al. ${ }^{16}$ proposed the use of color maps generated from closestpoint distances between the surfaces. The CMF tool calculates thousands of color-coded surface distances in millimeters between 3D models surface triangles at two different time points. The difference between the two surfaces at any location can be quantified. Isolines (contour line tool) are used to delineate surface changes for specific regions of interest. Teeth, condyles, articular fossa, mandibular ramus and corpus, maxilla, nose, cheeks, upper and lower lips, and chin, can be selected and analyzed (Figure 4). Treatment outcome changes are described not as absolute displacement but as displacements relative to the cranial base.

The quantitative changes are visualized using color maps. The color maps indicate inward (blue) or outward (red) displacement between overlaid structures. An absence of changes is indicated by the green color. For example, in mandibular advancement surgery, the forward chin and lower lip displacement would be shown in a red color code; in mandibular setback surgery lower lip and chin surfaces would be shown in a blue color code (Figure 5). The registration on the cranial method has been validated and used since 2005. 5,17 


\section{DIGITAL CASTS SUPERIMPOSITION (Figure 6)}

The advent of digital models represents many advantages for both clinicians and researchers. Digital models are readily available on the computer screen and do not need physical storage. Numerous validation studies have been conducted and it is known that digital models are similar to plaster models when used during diagnosis and treatment planning. ${ }^{18}$ Repeated measurements performed on plaster and digital models did not show clinically significant differences. ${ }^{19}$

The digital models of each dental arch are scanned independently and need to be related in space to represent the patient occlusion. The upper digital model needs to be registered to the lower one or vice versa. Many different methods have been developed for this purpose: visually assessing the plaster models' occlusion and matching their relative position in the virtual space; by scanning a wax bite, and registering the upper model to the upper side of the wax bite and the lower model to its lower side; by mounting the models in a bracket of known relative position, or by scanning the plaster models in occlusion and using that relative positional information to register the upper model to the lower one.

In order to assess changes due to treatment, growth or relapse digital models offer the possibility of registering and superimposing records from different time points. A stable structure is used as the registration area. Different authors have demonstrated the stability of the palatal rugae ${ }^{20-22}$ Rugae-based registration of digital models can be performed on a landmark-basis - or n-point registration - or on a surface-basis - or surface-to-surface registration.

Digital models can also be combined with other imaging modalities: they can be registered to CBCT images and be used during surgical planning and manufacturing of the surgical splints ${ }^{23}$ Recently Rangel FA reported the registration of digital models to threedimensional photos. ${ }^{24}$ These multimodal images could improve our diagnosis and treatment planning processes and eventually will become the clinical standard, enhancing treatments provided by different specialties including orthodontics, periodontics, prosthodontics and restorative dentistry

\section{D PHOTOGRAPHY SUPERIMPOSITION (Figure 7)}

Currently available software packages have tools for superimposition of 3D photographs on landmarks or surface based regions in the soft tissue, but soft tissue structures are not stable structures of reference for superimposition. Soft tissue surface appearance varies with weight gain or loss, growth, aging and variations of any individual facial posture and emotion expressions. Technologies such as 3-D photogrammetry ${ }^{25-26}$ and laser scanning ${ }^{27-28}$ of the face have been used for 3D soft tissue superimposition, but their major limitation has been the inability to standardize registration of the images over time. Current procedures to integrate $3 \mathrm{D}$ facial images have reported significant errors in head positioning ${ }^{27-28}$ and potential errors in facial expression have not been assessed. ${ }^{29}$ Problems that need to be overcome with 3D photograph superimposition include inadequate use of fiducials, head position in acquisition, soft tissue capture errors, and current use non-rigid registration deformation of soft tissue contours to allow matching of 3D photograph to CBCT soft tissues.

The variability of soft tissue surface has important consequences to the choice of approaches for adequate registration of longitudinal images. A stable reference for superimposition of images is required for a standardized record of the relationship between the soft tissue facial mask and the underlying skeletal and dental structures. Currently, CBCT technology allows the use of stable reference structures. 


\section{The Role of Future Investigations}

Analysis of 3D CBCT images is much more complex than analysis of 2D cephalometric radiographs. Superimposition on landmarks, the usual method for cephalometric analysis, is not satisfactory in analysis of 3D images. While landmark location in 2D is hampered by overlapping multiple structures, locating 3D landmarks on complex curving structures is significantly more difficult. With the method developed at UNC, a cranial base superimposition, based on the surface contours of the bone, overcomes the landmark problem. In essence, instead of 3 or 4 landmark points as in Figure 2, the superimposition is based on $\sim 300$ thousands of points. ${ }^{5}$

Regional superimposition in the cranial base does not completely define the movement of the mandible relative to the maxilla. ${ }^{2-6,9}$ The pioneer works of Baumrind et al, ${ }^{2}$ Bjork and Skieller, ${ }^{30}$ Ghafari et al ${ }^{4}$, Halazonetis ${ }^{31}$ and Johnston ${ }^{32}$ revealed that relative displacement of mandibular and maxillary skeletal and dental components is critical because the resulting information may differ from conclusions formulated from the cranial base superimposition. Future studies are needed to investigate the use of different regional superimposition areas. Currently, the methodology presented in this paper has been applied to clinical research in progress. However, these procedures are still time consuming and computing intensive making their application in routine clinical use difficult. Our research in progress has focused on making available a simplified analysis for deriving quantitative data from 3D images.

\section{Acknowledgments}

Supported by National Institute for Dental and Craniofacial Research (NIDCR) DE017727, DE018962, DE005215, Fundação de Auxilio à Pesquisa do Estado do Maranhão (FAPEMA) 128/06.

\section{References}

1. Baster M, Rosas A, O'Higgins P. Craniofacial levels and the morphological maturation of the human skull. J Anat. Nov; 2006 209(5):637-54. [PubMed: 17062021]

2. Baumrind S, Ben-Bassat Y, Bravo LA, Curry S, Korn EL. Partitioning the components of maxillary tooth displacement by the comparison of data from three cephalometric superimpositions. Angle Orthod. 1996; 66(2):111-24. [PubMed: 8712489]

3. Efstratiadis S, Baumrind S, Shofer F, Jacobsson-Hunt U, Laster L, Ghafari J. Evaluation of Class II treatment by cephalometric regional superimpositions versus conventional measurements. Am J Orthod Dentofac Orthop. Nov; 2005 128(5):607-18.

4. Ghafari J, Baumrind S, Efstratiadis SS. Misinterpreting growth and treatment outcome from serial cephaographs. Clin Orthod Res. Nov; 1998 1(2):102-6. [PubMed: 10321138]

5. Cevidanes LH, Bailey LJ, Tucker GR Jr, Styner MA, Mol A, Phillips CL, Proffit WR, Turvey T. Superimpositon of 3D cone-beam CT models of orthognathic surgery patients. Dentomaxillofac Radiol. 2005; 34(6):369-375. [PubMed: 16227481]

6. Cevidanes LH, Franco AA, Gerig G, Proffit WR, Slice DE, Enlow DH, Yamashita HK, Kim YJ, Scanavini MA, Vigorito JW. Assessment of mandibular growth and response to orthopedic treatment with 3-dimensional magnetic resonance images. Am J Orthod Dentofac Orthop. Jul; 2005 128(1):16-26.

7. Bookstein FL, Schafer K, Prossinger H, Seidler H, Fielder M, St ringer C, et al. Comparing frontal cranial profiles in archaic and modern homo by morphometric analysis. Anat Rec. 1999; 257:21724. [PubMed: 10620751]

8. Bookstein, FL. Morphometric tools for landmark data. 1st ed.. Cambridge University Press; Cambridge: 1991. p. 435

9. Cevidanes LH, Styner MA, Proffit WR. Image analysis and superimposition of 3-dimensional conebeam computed tomography models. Am J Orthod Dentofac Orthop. 2006; 129(5):611-618. 
10. Yushkevich PA, Piven J, Hazlett HC, Smith RG, Ho S, Gee JC, Gerig G. User-guided 3D active contour segmentation of anatomical structures: significantly improved efficiency and reliability. Neuroimage. Jul 1; 2006 31(3):1116-28. Epub 2006 Mar 20. [PubMed: 16545965]

11. Rueckert D, Sonoda LI, Hayes C, Hill DLG, Leach MO, Hawkes DJ. Nonrigid registrationusing free-form deformations: application to breast MR images. IEEE Trans Med Imaging. 1999; 18:712-21. [PubMed: 10534053]

12. Melson B. The cranial base. Acta Odontol Scand. 1974; 32(Suppl 62)

13. Chapuis J, Schramm A, Pappas I, Hallermann W, Schwenzer-Zimmerer K, Langlotz F, Caversaccio M. A new system for computer-aided preoperative planning and intraoperative navigation during corrective jaw surgery. IEEE Trans Inf Technol Biomed. May; 2007 11(3):27487. [PubMed: 17521077]

14. Andresen R, bookstein FL, Conradsen K, Ersboll BK, Marsh JL, Kreiborg S. Surface-bounded growth modeling applied to human mandibles. IEEE Trans Med Imaging. 2000; 19:1053-63. [PubMed: 11204843]

15. Mitteroecker P, Gunz P, Bookstein FL. Semilandmarks in three dimensions. Evol Dev. May-Jun; 2005 7(3):244-58. [PubMed: 15876197]

16. Gerig G, Jomier M, Chakos M. Valmet: a new validation tool for assessing and improving 3D object segmentation. Med Image Comput Comput Assist Interv Int Conf Med Image Comput Comput Assist Interv. 2001; 2208:516-528.

17. Cevidanes, LHS.; Styner, M.; Phillips, C.; Oliveira, AEF.; Tulloch, JFC. 3D morphometric changes 1 year after jaw surgery biomedical imaging: macro to nano; Proceedings of the IEEE International Symposium; Washington, DC. 2007; Apr:12-15; p. 1332-5.

18. Whetten JL, Williamson PC, Heo G, Varnhagen C, Major PW. Variations in orthodontic treatment planning decisions of Class II patients between virtual 3-dimensional models and traditional plaster models. Am J Orthod Dentofac Orthop. 2006; 130:485-491.

19. Stevens DR, Flores-Mir C, Nebbe B, Raboud DW, Heo G, Major PW. Validity, reliability, and reproducibility of paster vs digital study models: comparison of peer assessment ratiing and Bolton analysis and their constituent measurements. Am J Orthod Dentofac Orthop. 2006; 129:794-803.

20. Almeida MA, Phillips C, Kula K, Tulloch C. Stability of the palatal rugae as landmarks for analysis of dental casts. Angle Orthod. 1995; 65:43-48. [PubMed: 7726462]

21. Ashmore JL, Kurland BF, King GJ, Wheeler TT, Ghafari J, Ramsay DS. A 3-dimensional analysis of molar movement during headgear treatment. Am J Orthod Dentofac Orthop. 2002; 121:18-29. discussion 29-30.

22. van der Linden FP. Changes in the position of posterior teeth in relation to ruga points. Am J Orthod. 1978; 74:142-161. [PubMed: 278487]

23. Gateno J, Xia J, Teichgraeber JF, Rosen A, Hultgren B, Vadnais T. The precision of computergenerated surgical splints. J Oral Maxillofac Surg. 2003; 61:814-817. [PubMed: 12856256]

24. Rangel FA, Maal TJ, Berge SV, van Vlijmen OJ, Plooij JM, Schutyser F, et al. Integration of digital dental casts in 3-dimensional facial photographs. Am J Orthod Dentofac Orthop. 2008; 134:820-0826.

25. Ayoub AF, Xiao Y, Khambay B, Siebert JP, Hadley D. Towards building a photo-realistic virtual human face for craniomaxillofacial diagnosis and treatment planning. Int J Oral Maxillofac Surg. May; 2007 36(5):423-8. Epub 2007 Apr 10. [PubMed: 17428638]

26. Sawyer AR, See M, Nduka C. 3D Stereophotogrammetry Quantitative Lip Analysis. Asethetic Plast Surg. Jun 27.2008 Epub ahead of print.

27. Terai H, Shimahara M, Sakinaka Y, Tajima S. Accuracy of integration of dental casts in threedimensional models. J Oral Maxillofac Surg. 1999; 57:662-5. [PubMed: 10368089]

28. Nishii Y, Nojima K, Takane Y, et al. Integration of the maxillofacial three-dimensional CT image and the three-dimensional dental surface image. J Japan Orthod Soc. 1998; 57:189-94.

29. Hajeer, My; Millett, Dt; Ayoub, AF.; Siebert, JP. Applications of 3D imaging in orthodontics: part II. J orthod. Mar; 2004 31(1):155-62.

30. Bjork A, Skieller V. Normal and abnormal growth of the mandible. A systhesis of longitudinal cephalometric implant studies over a period of 25 years. Eur J Orthod. 1983; 5:1-46. [PubMed: 6572593] 
31. Halazonetis DJ. Computer-assisted cephalometric analysis. Am J Orthod Dentofac Orthop. 1994; 105:517-21.

32. Johnston LE Jr. Balancing the books on orthodontic treatment: An integrated analysis of change. Brit J Orthod. 1996; 23:93-102. [PubMed: 8771331] 


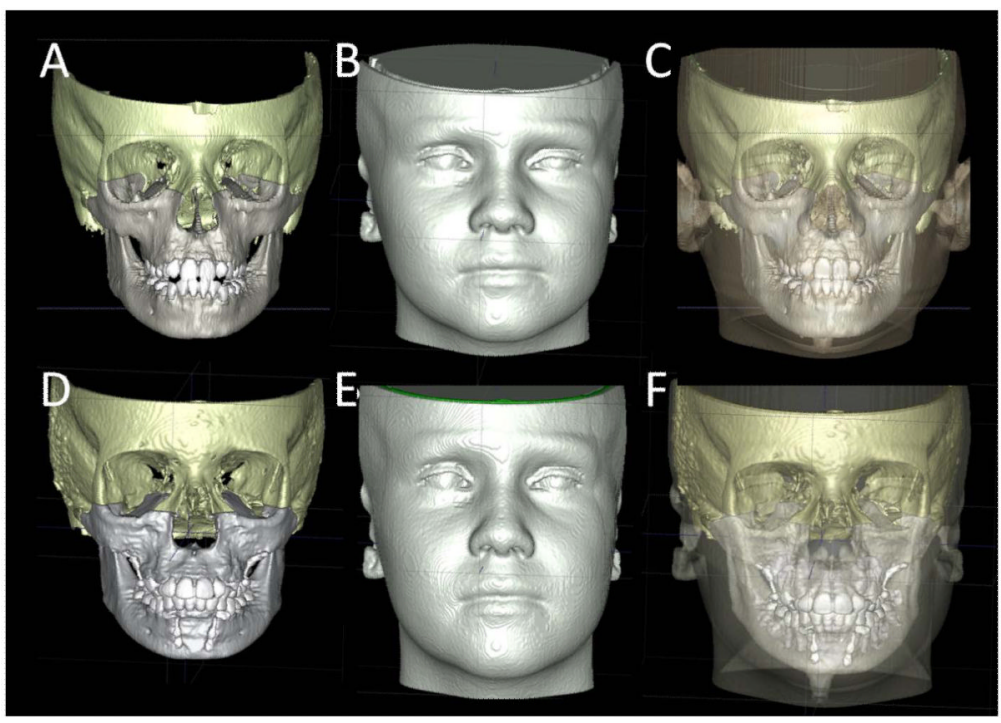

Figure 1.

Construction of 3D models from Cone-beam CT (CBCT) scans taken before and after Class III malocclusion orthopedic treatment with miniplates. . A, B and C, Pre-treatment models. A, Hard tissue. B, Soft tissue. C, Visualization of surface models with semi-transparency of soft tissues. (Color version of figure is available online.) 


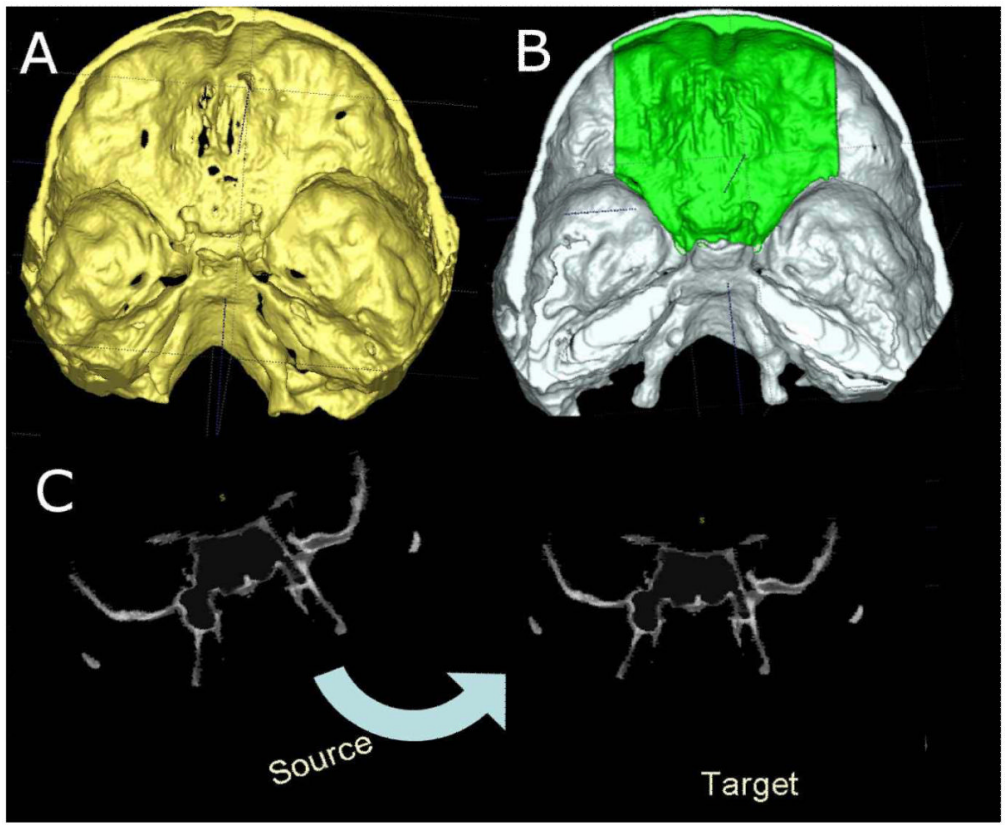

Figure 2.

A- Anatomic structures used to register 3D models of non-growing subjects in which we use the whole surface of the cranial base for registration of before and after treatment images. BAnatomic structures used to register 3D models of growing subjects in which we use the anterior surface of the cranial base for registration of before and after treatment images For growth assessment we have registered using the anterior cranial fossa surfaces, that have growth completed in early chidlhood, in such a way that the superimpositions describe growth relative to the individual cranial base. C- Fully automated calculation of rotational and translational parameters between the images. (Color version of figure is available online.) 


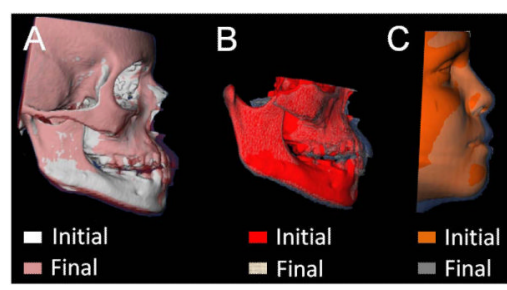

Figure 3.

Visualization of treatment changes with overlay of registered 3D surface models. A, B and $\mathrm{C}$ show different ways of visualization. A, Pre-treatment model (white) and post-treatment (semi-transparent red). B- Pre-treatment (red in the online version) and post-treatment (triangular mesh). The cranial base was cropped to show details of maxillo-mandibular changes. C, Soft tissue changes, pre-treatment (orange in the online version) and posttreatment (semi-transparent gray). (Color version of figure is available online.) 


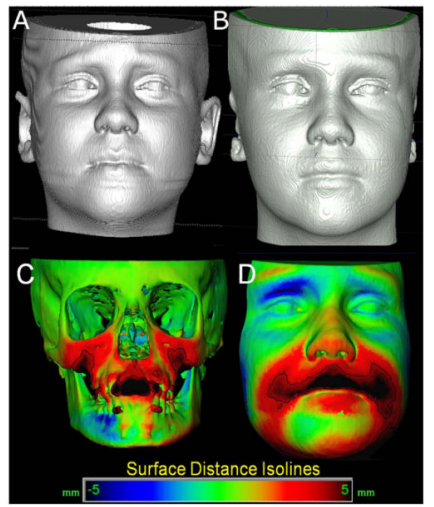

Figure 4.

Quantification of changes. A- Pre-treatment (white) and post-treatment 3D models (surface distance changes color map). Anterior displacement/remodeling is shown in red and posterior displacement/remodeling in blue. B- Color maps of hard and soft tissue regional changes. C- Isoline contours adjusted to quantify changes in the upper lip region. D- Isoline contours adjusted to quantify changes in the upper lip region. (Color version of figure is available online.) 


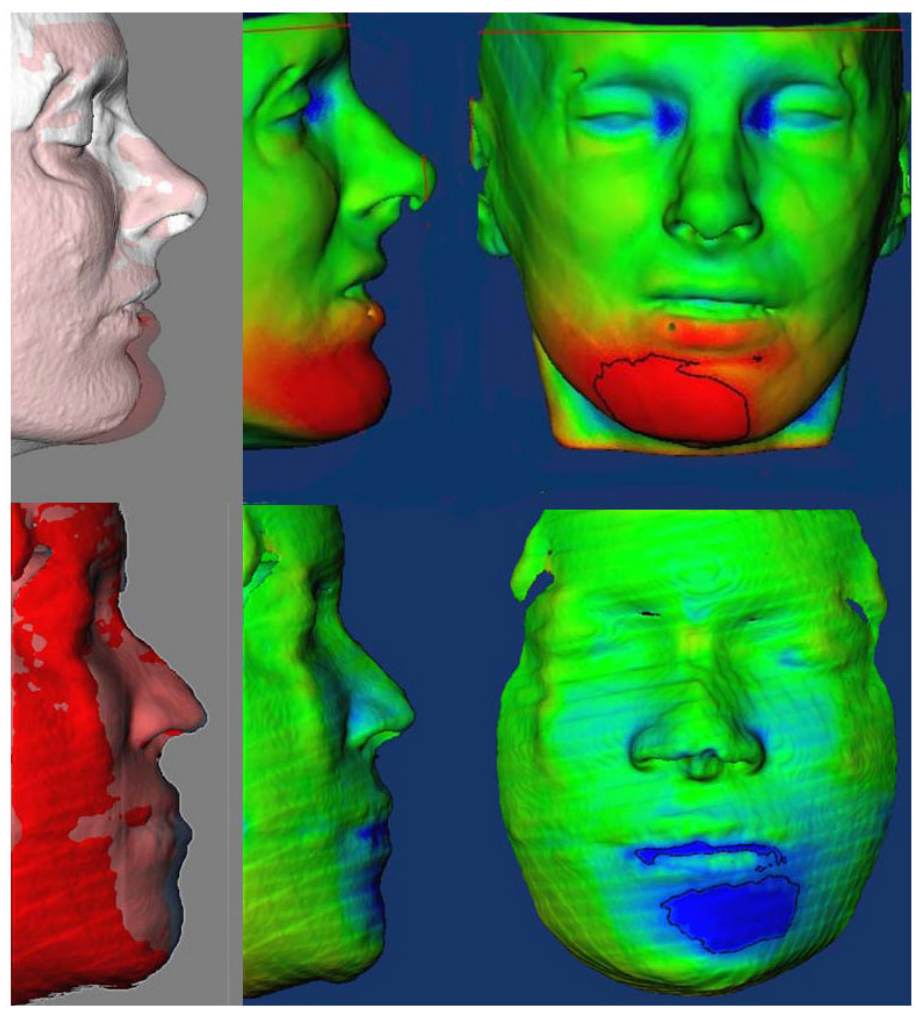

Figure 5 .

Soft tissue changes 1 year surgery. A, B and C, Mandibular advancement .D, E and F, Mandibular setback. A, Transparency overlays of superimposed pre-surgery (white) and 1 year post-surgery (red in the online version). $\mathbf{B}$ and $\mathbf{C}$, Surface distance color maps of soft tissue changes in the chin area. D, Transparency overlays of superimposed pre-surgery (red in the online version) and 1 year post-surgery (red in the online version). E and F, Surface distance color maps of soft tissue changes in the chin area. (Color version of figure is available online.) 


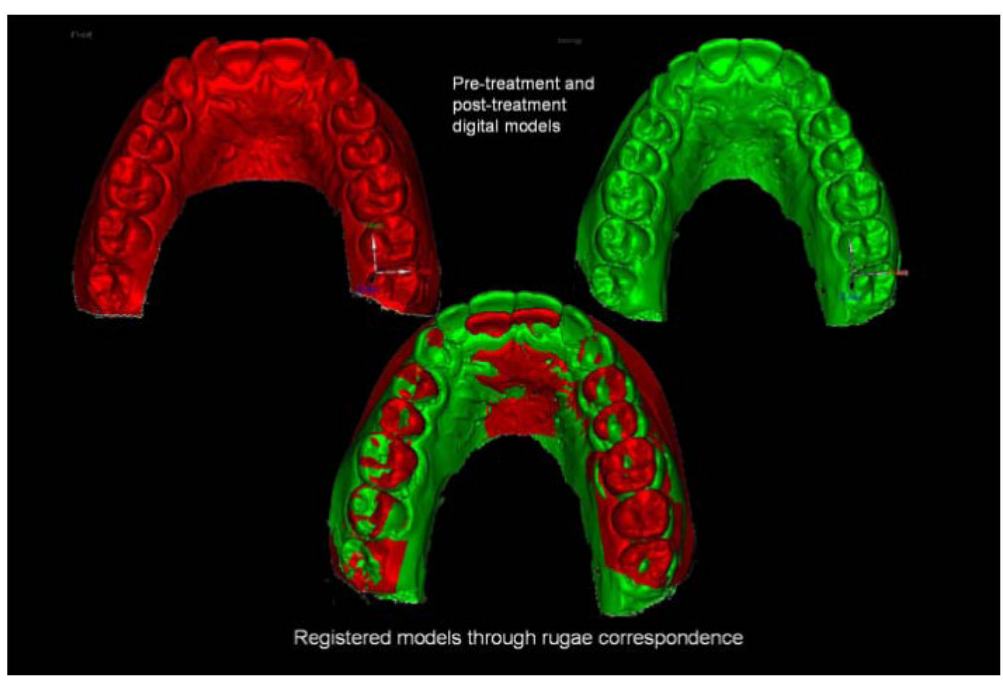

Figure 6.

Tooth movement changes. Registration of pre and post-treatment digital models using the palatal rugae. (Color version of figure is available online.) 


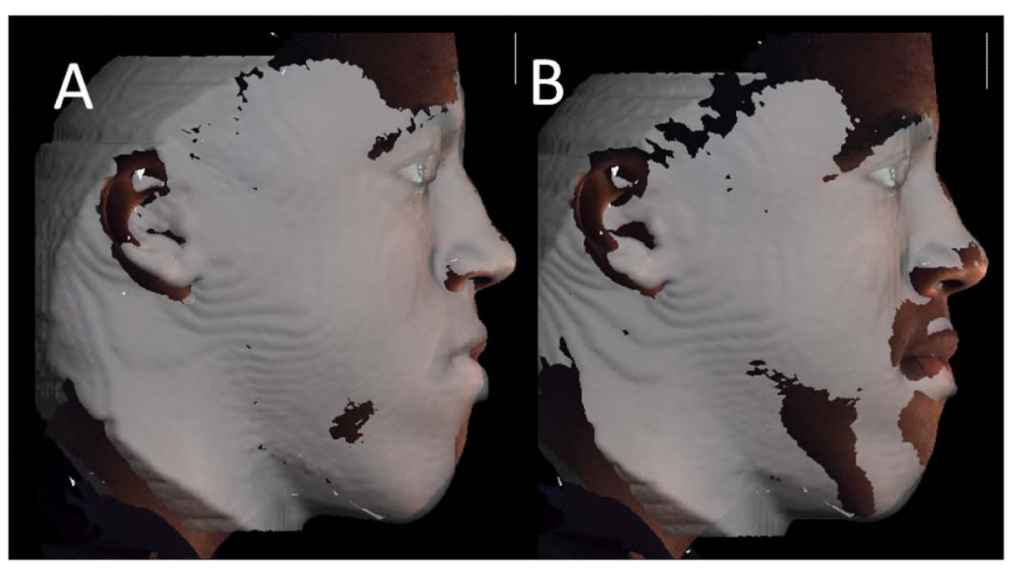

Figure 7.

Registration of patient 3D photograph to two CBCTs taken at the same day. A, First CBCT acquisition registered to 3D photograph. B, Second CBCT acquisition taken the same day. Both manual and soft tissue based registration were used to register the photograph and the CBCT surface model (3DMDVultus Software version 1.1, 3DMD Atlanta), the contours of the CBCT lower lip, chin and neck do not match the contours of the 3D photograph. (Color version of figure is available online.) 